\title{
Síndrome antifosfolipídico primario: características y evolución de una serie de 17 casos
}

\author{
J. Ma. CALVO ROMERO, J. C. BUREO DACAL, J. L. RAMOS SALADO, \\ P. BUREO DACAL, M. PÉREZ MIRANDA
}

Servicio de Medicina Interna. Hospital Regional Universitario Infanta Cristina. Badajoz.

\author{
PRIMARY ANTIPHOSPHOLIPID SYNDROME: CHARACTERISTICS \\ AND EVOLUTION OF A SERIES OF 17 CASES.
}

\section{RESUMEN}

Fundamento: El síndrome antifosfolipídico (SAF) primario es un trastorno trombofílico no asociado a colagenosis u otras enfermedades.

Método: Revisión retrospectiva de los pacientes diagnosticados de SAF primario en un servicio de Medicina Interna entre enero de 1996 y diciembre de 2000.

Resultados: Identificamos 17 pacientes con SAF primario. Las formas de presentación más frecuentes fueron la trombosis venosa profunda ( 7 casos) y el ictus isquémico ( 3 casos). Once pacientes $(65 \%)$ tuvieron trombosis venosa, 4 (24\%) trombosis arterial y ningún paciente tuvo ambos tipos de trombosis. Los 15 pacientes que presentaron trombosis recibieron tratamiento con anticoagulación oral indefinida (INR entre 2,5 y 3,5$)$. No hubo ninguna recurrencia de trombosis ni complicaciones hemorrágicas graves tras un tiempo medio de seguimiento de 36 meses (rango 10-52 meses).

Conclusiones: La forma de presentación más frecuente del SAF primario es la trombosis venosa profunda. La trombosis venosa es más frecuente que la trombosis arterial. La ocurrencia de ambos tipos de trombosis parece ser excepcional. La anticoagulación oral a largo plazo es un tratamiento eficaz y seguro para prevenir la recurrencia de trombosis en los pacientes con SAF primario.

PALABRAS CLAVE: Síndrome antifosfolipídico. Trombosis. Anticoagulación oral.

\begin{abstract}
Background: Primary antiphospholipid syndrome (APS) is a throm bophilic disorder which is not associated with connective tissue diseases or with other diseases.

Method: Retrospective review of patients diagnosed of primary APS in an Internal Medicine service between January 1996 and December 2000.

Results: We identified 17 patients with primary APS. The most com mon presenting manifestations were deep vein thrombosis ( 7 cases) and ischemic stroke ( 3 cases). Eleven patients (65\%) had vein thrombosis, 4 (24\%) arterial thrombosis and no patient had both types of thrombosis. The 15 patients with thrombosis received treatment with indefinite oral anticoagulation (INR between 2.5 and 3.5). There was no recurrence of thrombosis or major hemorrhagic complications after a median followup time of 36 months (range 10-52 months).

Conclusions: The most common presenting manifestation of primary APS is deep vein thrombosis. Venous thrombosis is more frequent than arterial thrombosis. The occurrence of both types of thrombosis seems to be exceptional. Long-term oral anticoagulation is an effective and safe treatment to prevent the recurrence of thrombosis in patients with pri mary APS.
\end{abstract}

KEY WORDS: Antiphospholipid syndrome. Thrombosis. Oral anticoa gulation.

Calvo Romero JMa , Bureo Dacal JC, Ramos Salado JL, Bureo Dacal P, Pérez. Miranda M. Síndrome antifosfolipídico primario: caracterís ticas y evolución de una serie de 17 casos. An Med Interna (Madrid) 2002; 19: 226-229.

\section{INTRODUCCIÓN}

El síndrome antifosfolipídico (SAF), también denominado síndrome de los anticuerpos antifosfolípidos (AAF) o síndrome de Hughes, es un trastorno trombofílico que se asocia a trombosis venosas, trombosis arteriales y abortos de repetición (1-3). Los marcadores serológicos del SAF son los anticuerpos anticardiolipina (AAC) y el anticoagulante lúpico (AL), que se incluyen bajo la denominación de AAF (1-3).
En los primeros años de la década de los 80 se comunicaron los primeros casos de enfermedad tromboembólica venosa, pérdidas fetales recurrentes y trombopenia en pacientes con AL y lupus eritematoso sistémico (LES) (1). Posteriormente se describió el SAF en pacientes con otras colagenois y en pacientes que no tenían un LES ni otras enfermedades asociadas, estableciéndose el concepto de SAF primario (4-6). En los últimos años ha habido un creciente interés en el estudio del SAF, debido a su implicación en enfermedades con una alta prevalencia, como la enfermedad tromboembólica veno-

Trabajo aceptado: 30 de enero de 2002

Correspondencia: José María Calvo Romero. Héroes de Cascorro, $93^{\circ}$ A. 06004 Badajoz. 
sa, los ictus isquémicos especialmente en menores de 50 años y los abortos de repetición (1-3). No obstante, todavía el SAF y especialmente el SAF primario no es una entidad perfectamente caracterizada en diversos aspectos. En el presente trabajo revisamos las características y evolución de nuestra serie de pacientes con SAF primario.

\section{PACIENTES Y MÉTODOS}

Revisión retrospectiva de los pacientes diagnosticados de SAF primario en nuestro servicio de Medicina Interna entre enero de 1996 y diciembre de 2000. El diagnóstico de SAF se estableció según los criterios definidos en el International Consen sus Statement on Preliminary Classification Criteria for Definite Antiphospholipid Syndrome (Sapporo 1998) (7). El diagnóstico de SAF primario se estableció siguiendo los criterios de exclusión de Piette y cols. (8) (Tabla I), si bien no se exigió un tiempo de seguimiento superior a 5 años. Se estudiaron en el momento del diagnóstico las características epidemiológicas y clínicas y los siguientes datos analíticos: recuento plaquetario, tiempo de tromboplastina parcial activado (TTPA), títulos de AAC IgG e IgM y AL. Los AAC se determinaron mediante la técnica de ELISA (enzyme-linked immunosorbent assay). Se consideró positivo un título de AAC IgG igual o superior a 15 GPL y/o un título de AAC IgM igual o superior a 15 MPL. Los títulos reflejados de AAC corresponden a la media aritmética de las 2 primeras determinaciones. El anticoagulante lúpico se determinó según las directrices del Subcommitee for the Stan dardization of Lupus Anticoagulants of the International Society of Thrombosis and Hemostasis (9). Todos los episodios de trombosis venosa y arterial fueron diagnosticados mediante pruebas objetivas. Igualmente se registró el tratamiento instaurado y la ocurrencia de recurrencias de trombosis y complicaciones hemorrágicas graves. Se consideraron complicaciones hemorrágicas graves las que precisaron ingreso hospitalario.

\section{TABLA I}

\section{CRITERIOS DE EXCLUSIÓN DE SAF PRIM ARIO (8)}

\section{Eritema malar}

Lupus discoide

Úlceras orofaríngeas

Artritis franca

Pleuritis (en ausencia de embolismo pulmonar o fallo cardíaco)

Pericarditis (en ausencia de infarto agudo de miocardio o uremia)

Proteinuria persistente $>500 \mathrm{mg}$ / día debida a glomerulonefritis mediada por inmunocomplejos

Linfopenia $<1000 / \mathrm{mm}^{3}$

Anticuerpos anti-DNA y/ 0 anti-ENA

Anticuerpos antinucleares $>1: 320$

Tratamiento con drogas que inducen anticuerpos antifosfolípidos

SAF: síndrome antifosfolipídico.

Las variables continuas se expresan como media \pm desviación standard. El análisis estadístico se realizó mediante el programa EPI-INFO 5,01 utilizando la prueba chi cuadrado y el test exacto de Fisher, cuando alguno de los valores esperados fue menor de 5, para la comparación de proporciones y la prueba de la "t" de Student para la comparación de medias. Se consideró estadísticamente significativo un valor de $\mathrm{p}<0,05$.

\section{RESULTADOS}

Identificamos 17 pacientes con SAF primario. Doce $(71 \%)$ fueron varones. La edad media en el momento del diagnóstico fue 38,1 $\pm 10,8$ años (rango 19-60 años). Las formas de presentación fueron: trombosis venosa profunda (TVP) de miembros inferiores en 6 casos (35\%) con tromboembolismo pulmonar en uno de ellos, TVP de miembros superiores en 1 caso (6\%), ictus isquémico en 3 casos (18\%), abortos de repetición en 2 casos (12\%), síndrome de Budd-Chiari en 1 caso $(6 \%)$, trombosis venosa mesentérica en 1 caso $(6 \%)$, trombosis portal en 1 caso $(6 \%)$, isquemia arterial aguda de miembros inferiores en 1 caso $(6 \%)$, y trombosis venosa suprarrenal en 1 caso $(6 \%)$. Once pacientes $(65 \%)$ presentaron trombosis venosa, 4 (24\%) trombosis arterial y ningún paciente presentó ambos tipos de trombosis. En la tabla II se comparan las características de los pacientes con trombosis venosa y con trombosis arterial. Sólo 2 pacientes tuvieron un evento trombótico (una TVP de miembros inferiores y una trombosis venosa esplenoportal) previo al que ocurrió en el momento del diagnóstico.

Dos pacientes (12\%) tuvieron trombopenia (recuento plaquetario $<140000 / \mathrm{mm}^{3}$ ) en el momento del diagnóstico o durante el periodo estudiado. Once pacientes $(65 \%)$ tuvieron un TTPA prolongado (>38 segundos) en el momento del diagnóstico. Seis pacientes $(35 \%)$ tuvieron AAC IgG positivos, y $6(35 \%)$ AAC IgM positivos, y el título medio fue $46,4 \pm 6,3$ GPL y 27,8 $\pm 5,1 \mathrm{MPL}$, respectivamente. Catorce pacientes $(82 \%)$ tuvieron un AL positivo en el momento del diagnóstico. Seis pacientes (35\%) tuvieron sólo AL positivo, 3 (18\%) sólo AAC positivos y 8 (47\%) tanto AL como AAC positivos. Cuatro pacientes (29\%) del total de 14 con AL positivo tuvieron un TTPA normal.

El tiempo medio de seguimiento fue de 36 meses (rango 10-52 meses). Los 15 pacientes que presentaron trombosis recibieron tratamiento con anticoagulación oral indefinida (INR entre 2,5 y 3,5). Las 2 pacientes con abortos de repetición recibieron tratamiento con ácido acetilsalicílico (300 mg diarios). No hubo ninguna recurrencia de trombosis ni complicaciones hemorrágicas graves en nuestros pacientes durante el periodo estudiado. Ningún paciente cumplió criterios de LES u otra colagenosis a lo largo del periodo estudiado.

\section{DISCUSIÓN}

Nuestra serie incluye pacientes que cumplen los nuevos criterios diagnósticos de SAF establecidos en el Consenso Internacional celebrado en Sapporo en 1998 (7). Estos criterios exigen la ocurrencia de trombosis y/o abortos de repetición para establecer el diagnóstico de SAF, excluyendo la trombopenia como criterio diagnóstico. Un tanto por ciento no despreciable de los casos de series previamente publicadas de SAF primario debería ser excluido en la actualidad. Se ha recomendado un tiempo de seguimiento de al menos 5 años antes de establecer el diagnóstico de SAF primario, fundamentalmente por la posibilidad de que algunos casos acaben cumpliendo criterios de LES (8). No obstante, la evolución del SAF primario a LES es excepcional (10). Además, parece que el grupo de pacientes con SAF que no puede ser incluido dentro del SAF primario ni del SAF secundario a LES presenta características más similares al SAF primario (11). 
En nuestra serie, el SAF primario ocurrió más frecuentemente en varones a diferencia de otras series, en las que existe un predominio del sexo femenino (4,12-14). Posiblemente, esto sea debido al menos en parte al escaso número de mujeres con abortos de repetición como única manifestación clínica del SAF primario en nuestro estudio. La edad media en el momento de la presentación de nuestros pacientes fue bastante similar a la de otras series, situándose entre los 30 y 40 años (4,12-14).

La forma de presentación más frecuente en nuestros pacientes con SAF primario fue la trombosis venosa profunda, y la forma de trombosis arterial más frecuente fue el ictus isquémico, al igual que en otras series $(4,12-14)$. No obstante, nuestro estudio ilustra la gran variedad de localizaciones de trombosis venosa y arterial posibles en el SAF primario, y la necesidad de mantener un alto índice de sospecha ante cualquier evento trombótico no explicado. Por ejemplo, como ocurrió en nuestra serie, el síndrome de Budd-Chiari y la insuficiencia suprarrenal primaria son posibles manifestaciones del SAF primario $(15,16)$.

La trombosis venosa y la trombosis arterial no coexistieron en ninguno de nuestros pacientes. En las series de Asherson y cols. (4) y Barquinero y cols. (13), sólo un $14 \%$ y un $15 \%$ respectivamente de los pacientes con SAF primario tuvieron ambos tipos de trombosis. Una hipótesis para explicar este hallazgo sería que en el desarrollo de la trombosis arterial y de la trombosis venosa en el SAF primario influyeran distintos factores etiopatogénicos. No obstante, factores de riesgo de ateroesclerosis y por tanto de trombosis arterial como la hipertensión arterial, la diabetes mellitus, la hiperlipemia y el tabaquismo no fueron más frecuentes en nuestros pacientes con trombosis arterial que en los pacientes con trombosis venosa (Tabla II), lo que sugiere que estos factores de riesgo pueden no tener un papel relevante en la etiopatogenia de la trombosis arterial en los pacientes con SAF primario. Además no encontramos diferencias en las características estudiadas entre nuestros pacientes con trombosis venosa y arterial (Tabla II). Queda pues por dilucidar si la ocurrencia de trombosis venosa o arterial está influenciada por factores diferentes.

La frecuencia descrita de positividad del AL y de los AAC en los pacientes con SAF primario es muy variable (4,12-14), posiblemente debido a cuestiones metodológicas. Quizás más relevante resulta que algunos pacientes con SAF primario sólo presentan positividad del AL o de los AAC (4), como se confirma en nuestra serie. Esto obliga a determinar en todos los pacientes con sospecha de SAF primario tanto el AL como los
TABLA II

COM PARACIÓ N DE LAS CARACTERÍSTICAS DE LOS

PACIENTES CON TROM BO SIS VENOSA Y ARTERIAL

\begin{tabular}{lccc}
\hline & $\begin{array}{c}\text { TV } \\
n=11\end{array}$ & $\begin{array}{c}\text { TA } \\
n=4\end{array}$ & $p$ \\
\hline Edad (años) & $40,2 \pm 11,6$ & $34,7 \pm 10,9$ & NS \\
Sexo varón & $8(73 \%)$ & $4(100 \%)$ & NS \\
Hipertensión arterial & $2(18 \%)$ & $1(25 \%)$ & NS \\
Diabetes mellitus & 0 & 0 & NS \\
Hiperlipemia & $2(18 \%)$ & $1(25 \%)$ & NS \\
Tabaquismo & $8(73 \%)$ & $2(50 \%)$ & NS \\
Trombopenia (<140000 mm $\left.^{3}\right)$ & $2(18 \%)$ & 0 & NS \\
TTPA alargado (>38 segundos) & $7(64 \%)$ & $3(75 \%)$ & NS \\
TTPA (segundos) & $41,7 \pm 13,6$ & $40,8 \pm 8,3$ & NS \\
AAC IgG positivo & $4(36 \%)$ & $1(25 \%)$ & NS \\
AAC IgM positivo & $4(36 \%)$ & $1(25 \%)$ & NS \\
AL positivo & $10(91 \%)$ & $4(100 \%)$ & NS \\
\hline
\end{tabular}

TV: trombosis venosa. TA: trombosis arterial. TTPA: tiempo de tromboplastina parcial activada. AAC: anticuerpos anticardiolipina. AL: anticoagulante lúpico. NS: no significación estadística $(p \geq 0,05)$.

AAC. Aunque la presencia de AL se asocia a una prolongación del TTPA (1-3), una parte de los pacientes con AL puede tener un TTPA normal, como ocurrió en nuestro estudio. La trombopenia en el momento del diagnóstico o en el seguimiento fue menos frecuente en nuestros pacientes con SAF primario que en los de otras series, posiblemente porque como se ha comentado anteriormente la trombopenia ha sido excluida de los nuevos criterios diagnósticos de SAF (7) utilizados en nuestro estudio.

La anticoagulación oral a largo plazo es el tratamiento recomendado para prevenir las recurrencias de trombosis en los pacientes con SAF (1-3). Existe controversia en cuanto al nivel más adecuado de la anticoagulación oral. Todos nuestros pacientes con trombosis fueron tratados con anticoagulación oral indefinida (INR entre 2,5 y 3,5), y no hubo ninguna recurrencia de trombosis ni complicaciones hemorrágicas graves durante un periodo medio de seguimiento de 36 meses. Así pues, la anticoagulación oral indefinida parece ser un tratamiento eficaz y seguro para prevenir la recurrencia de trombosis en los pacientes con SAF primario.

\section{Bibliografía}

1. Hughes GRV. The antiphospholipid syndrome: ten years on. Lancet 1993; 342: 341-4.

2. Khamashta MA, Hughes GRV. Antiphospholipid antibodies and antiphospholipid syndrome. Curr Opin Rheumatol 1995; 7: 389-394.

3. Calvo Romero JM. Síndrome antifosfolípido. Revista Clínica Española 1999; 199: 596-602.

4. Asherson RA, Khamashta MA, Ordi-Ros J, Derksen RHWM, Machin SJ, Barquinero J et al. The "primary" antiphospholipid syndrome: major clinical and selogical features. Medicine (Baltimore) 1989; 68: 366-74.

5. Mackworth-Young CG, Loizou S, Walport MJ. Primary antiphospholipid syndrome: features of patients with raised anticardiolipin antibodies and no other disorder. Ann Rheum Dis 1989; 48: 362-7.

6. Alarcón-Segovia D, Sánchez-Guerrero J. Primary antiphospholipid syndrome. J Rheumatol 1989; 16: 482-8.

7. Wilson WA, Gharavi AE, Koike T, Lockshin MD, Branch DW, Piette JC et al. International Consensus Statement on Preliminary Classification Criteria for Definite Antiphospholipid Syndrome: report of an international workshop. Arthritis Rheum 1999; 42: 1309-11.

8. Piette JC, Wechsler B, Frances C, Papo T, Godeau P. Exclusion criteria for primary antiphospholipid syndrome. J Rheumatol 1993; 20: 1802-3.

9. Brandt JT, Barna LK, Triplett DA. Laboratory identification of lupus anticoagulants: results of the Second International Workshop for Identification of Lupus anticoagulants. Thromb Haemost 1995; 74: 1597-603. 
10. Blanco Y, Ramos-Casals M, García-Carrasco M, Cervera R, Ingelmo M. Síndrome antifosfolipídico que evoluciona a lupus eritematoso sistémico: presentación de tres nuevos casos y revisión de la literatura. Rev Clin Esp 1999; 199: 586-8.

11. Weber M, Hayem G, De Bandt M, Seifert B, Palazzo E, Roux S et al. Classification of an intermediate group of patients with antiphospholipid syndrome and lupus-like disease: primary or secondary antiphospholipid syndrome. J Rheumatol 1999; 26: 2131-6.

12. Font J, Lopez-Soto A, Cervera R, Balasch J, Pallares L, Navarro M et al. The "primary" antiphospholipid syndrome: antiphospholipid antibody pattern and clinical features of a series of 23 patients. Autoimmunitiy 1991; 9: 69-75.
13. Barquinero J, Ordi J, Vilardell M, Jordana R, Tolosa C, Selva A et al. Síndrome antifosfolípido primario: estudio de 27 pacientes. Med Clin (Barc) 1990; 94: 41-5.

14. Martínez-Vázquez C, Albo C, Rivera A, Bordón J, Rodríguez A, Sopeña B et al. Síndrome antifosfolípido primario. Aspectos clínico-evolutivos de 24 casos. Rev Clin Esp 1994; 194: 164-9.

15. Garrido JA, Díaz Peromingo JA, Martínez-Deben FS, Grandes J. Síndrome de Budd-Chiari como forma de presentación de un síndrome antifosfolípido primario. An Med Interna (Madrid) 1996; 13: 355-6.

16. Molina M, Ortega G, Bermudo J, Cuesta P. Insuficiencia suprarrenal asociada a síndrome antifosfolípido primario. An Med Interna (Madrid) 1995; 12: 232-4. 